Check for updates

Cite this: Mater. Horiz., 2020, 7,592

Received 13th August 2019,

Accepted 31st October 2019

DOI: $10.1039 / \mathrm{c} 9 \mathrm{mh} 01261 \mathrm{c}$

rsc.li/materials-horizons

\section{High- $\kappa$ polymers of intrinsic microporosity: a new class of high temperature and low loss dielectrics for printed electronics $\dagger$}

\author{
Zhongbo Zhang, (D) $\ddagger^{a}$ Jifu Zheng, (D) $\ddagger^{b}$ Kasun Premasiri, (D) $\ddagger^{c}$ Man-Hin Kwok, ${ }^{a}$ \\ Qiong Li, ${ }^{a}$ Ruipeng Li, (D) ${ }^{d}$ Suobo Zhang, (D) ${ }^{\text {b }}$ Morton H. Litt, ${ }^{a}$ Xuan P. A. Gao (D) \\ and Lei Zhu (D) *a
}

High performance polymer dielectrics are a key component for printed electronics. In this work, organo-soluble polymers of intrinsic microporosity (PIMs) are reported for the first time to demonstrate desirable dielectric properties with a high permittivity (or $\kappa$ ), heat resistance, and low dielectric loss simultaneously. Due to the highly dipolar sulfonyl side groups $(4.5 \mathrm{D})$ and rigid contorted polymer backbone, a sulfonylated PIM ( $\mathrm{SO}_{2}-\mathrm{PIM}$ ) enabled frictionfree rotation of sulfonyl dipoles in the nanopores. As such, an optimal balance between relatively high $\kappa$ and low dielectric loss is achieved in a broad temperature window $\left(-50-200{ }^{\circ} \mathrm{C}\right)$. For example, the discharged energy density reached $17 \mathrm{~J} \mathrm{~cm}^{-3}$ with $\kappa$ $=6.0$. The discharge efficiency was $94 \%$ at $150{ }^{\circ} \mathrm{C} / 300 \mathrm{MV} \mathrm{m}^{-1}$ and $88 \%$ at $200{ }^{\circ} \mathrm{C} / 200 \mathrm{MV} \mathrm{m}^{-1}$. Furthermore, its application as a highgate dielectric in field effect transistors (FETs) is demonstrated. With the bilayer $\mathrm{SO}_{2}-\mathrm{PIM} / \mathrm{SiO}_{2}$ gate dielectric, InSe FETs exhibited a high electron mobility in the range of $200-400 \mathrm{~cm}^{2} \mathrm{~V}^{-1} \mathrm{~s}^{-1}$, as compared to $40 \mathrm{~cm}^{2} \mathrm{~V}^{-1} \mathrm{~s}^{-1}$ for the bare $\mathrm{SiO}_{2}$-gated InSe FET. This study indicates that highly dipolar PIMs with a rigid polymer backbone and large free volume are promising as next generation gate dielectric materials for printed electronics.

High-dielectric constant (or high- $\kappa$ ) and low-loss polymer dielectrics are highly desirable for power and electronic applications, including electric energy storage $\mathrm{e}^{1-3}$ and printed electronics $^{4,5}$ such as in human performance monitoring, ${ }^{6}$ information storage devices, ${ }^{7}$ and soft robotics. ${ }^{8}$ One important

\footnotetext{
${ }^{a}$ Department of Macromolecular Science and Engineering, Case Western Reserve University, 2100 Adelbert Road, 44106-7202, USA. E-mail: lxz121@case.edu

${ }^{b}$ Key Laboratory of Ecomaterials, Changchun Institute of Applied Chemistry, Chinese Academy of Sciences, Changchun 130022, P. R. China

${ }^{c}$ Department of Physics, Case Western Reserve University, 2076 Adelbert Road, Cleveland, Ohio 44106, USA

${ }^{d}$ National Synchrotron Light Source II, Brookhaven National Laboratory, Upton, New York 11973, USA

$\dagger$ Electronic supplementary information (ESI) available. See DOI: 10.1039/ c9mh01261c

\$ These authors contributed equally to this work and should be considered as co-first authors.
}

\section{New concepts}

In this work, a new concept of a friction-free dipolar glass polymer is demonstrated using novel sulfonylated polymers of intrinsic microporosity ( $\mathrm{SO}_{2}$-PIMs). First, the nanopores in PIMs provide large free volume for friction-free rotation of side-chain dipolar groups. Second, the sulfonyl group has a large dipole moment of $4.5 \mathrm{D}$ to promote orientational polarization. Third, the rigid, contorted, ladder-like backbone of PIM enables a high glass transition temperature $\left(T_{\mathrm{g}}\right.$, above the thermal degradation temperature). As a result of friction-free rotation of sulfonyl dipoles in the nanopores below the $T_{\mathrm{g}}, \mathrm{SO}_{2}$-PIM shows a dissipation factor as low as 0.005 , while achieving a high dielectric constant $(\kappa=6)$ and a high discharged energy density of $17 \mathrm{~J} \mathrm{~cm}^{-3}$ with discharge efficiency $>90 \%$ at room temperature. Even at $175{ }^{\circ} \mathrm{C}$, the discharge efficiency maintained at $86 \%$, indicating excellent heat tolerance. Compared to existing high $\kappa$ dipolar polymers, this concept has never been demonstrated before. Furthermore, the dielectric performance of $\mathrm{SO}_{2}$-PIM is tested in the InSe field-effect transistors (FETs). Intriguingly, the high $\kappa \mathrm{SO}_{2}$-PIM significantly promotes the electron mobility in InSe to $200-400 \mathrm{~cm}^{2} \mathrm{~V}^{-1} \mathrm{~s}^{-1}$. This work demonstrates that $\mathrm{SO}_{2}$-PIMs are promising as next generation gate dielectric materials for printed electronics.

component for all hybrid electronic devices is the organic field effect transistor (FET), where high- $\kappa /$ low-loss gate dielectrics play an important role. ${ }^{9-12}$ The state-of-the-art gate dielectric used in industry is self-assembled monolayer (SAMs, $\sim 2 \mathrm{~nm}$ ) passivated silica $\left(\mathrm{SiO}_{2}\right)$ or silicon oxynitride, which can induce high hole mobility $\left(\mu_{\mathrm{h}}\right)$ for semiconductors. ${ }^{13}$ However, $\mathrm{SiO}_{2}$ has a relatively low $\kappa$ of 3.9, and the leakage current increases exponentially as its thickness decreases to below $50 \mathrm{~nm}$ due to Fowler-Nordheim electron tunneling. ${ }^{14,15}$ It has been a challenge to search for high- $\kappa /$ low-loss printable dielectrics that can fulfill the stringent requirements for organic FETs, including but not limited to, $\kappa>5-10$, capacitance $>20 \mathrm{nF} \mathrm{cm}^{-2}$, low leakage, high breakdown strength, and long lifetime.

Theoretically, the capacitance density $\left(C_{g}\right.$, in $\left.\mathrm{F} \mathrm{m}^{-2}\right)$ of a gate dielectric is defined as: $C_{\mathrm{g}}=\varepsilon_{0}(\kappa / d)$, where $\varepsilon_{0}$ is vacuum permittivity and $d$ is thickness of the effective layer. Fundamentally, there are two ways to achieve high capacitance density: decreasing $d$ and/or increasing $\kappa$. In the past three decades, 
research has been focused on decreasing $d$, rather than increasing $\kappa$, because it has been difficult to increase $\kappa$ above 5 for linear dielectric polymers. ${ }^{1,16}$ For example, neat SAMs (2-4 nm thick) and ultrathin polymer films $(15-20 \mathrm{~nm})$ have been reported to show a high capacitance density, i.e., $>300 \mathrm{nF} \mathrm{cm}{ }^{-2} \cdot{ }^{13}$ However, manufacturing defects in SAMs, as well as ultrathin polymer films, limits the device size to $0.1 \mathrm{~mm}^{2}$ (e.g., both molecular and mesoscale pinholes and grain boundaries are present in $\mathrm{SAMs}^{17}$ ), despite decades of research to push for molecularly thin dielectrics. ${ }^{18}$ Grand challenges exist to achieving defect-free molecular dielectrics using currently available manufacturing processes. $^{18}$

Given the recent advances, ${ }^{1,2}$ an alternative approach to achieving high performance organic FETs is to increase the permittivity of the polymer dielectric above 5-10 while keeping low dielectric loss. In this way, relatively thick polymer films (0.3-1.0 $\mu \mathrm{m})$ can be printed or spin-coated, which will decrease the pinhole density while a high $C_{\mathrm{g}}$ can still be obtained via the high $\kappa$ gate dielectric. The first attempt is to utilize high $\kappa$ poly(vinylidene fluoride-co-trifluoroethylene) $[\mathrm{P}(\mathrm{VDF}-\mathrm{TrFE})]$-based random copolymers and terpolymers $(\kappa=50-70) .{ }^{19}$ These high $\kappa$ fluoropolymers have been applied for organic FETs. ${ }^{20,21}$ However, a high dissipation factor $(\tan \delta)$ of $\geq 0.02$ is observed, and the applicable frequency is limited at $<10^{4} \mathrm{~Hz}$ due to the slow switching of ferroelectric domains.

The second strategy is to utilize the space charge polarization (i.e., conduction of ions). Based on the electric double layer (EDL) theory, ${ }^{22}$ the capacitance from EDL can be at least 1000 times that of the dipolar capacitance of bulk polymers. Recently, ion gel gate dielectrics have been developed, which exhibit ultrahigh capacitance density, around $3-100 \mu \mathrm{F} \mathrm{cm}^{-2}$ at $1 \mathrm{~Hz}^{23}$ However, the operation frequency is limited at $<10^{4} \mathrm{~Hz}$ due to slow ion motion. The $\tan \delta$ is around 0.09-0.20 for EDL capacitors, ${ }^{23}$ too high for many practical FET applications.

Recently, dipolar glass (DG) polymers have attracted substantial attention because of their high $\kappa$ and low loss below the glass transition temperaure $\left(T_{\mathrm{g}}\right)$. Basically, DG polymers utilize the sub- $T_{\mathrm{g}}$ transitions to enhance orientational polarization. ${ }^{1,24}$ Most of these DG polymers contain cyanoethyl side groups (3.9 D). ${ }^{25-27}$ For example, Shin-Etsu Chemical commercialized cyanoethylated pullulan (CEP, 90\% functionality). Because of the relatively rigid cellulose backbone, the $T_{\mathrm{g}}$ reached $\sim 110{ }^{\circ} \mathrm{C},{ }^{28}$ and the room temperature permittivity was $\sim 16$ at frequencies below $10^{4} \mathrm{~Hz}^{29}$ However, CEP suffers from relativley high loss $(\tan \delta>0.02)$ and low breakdown strength $\left(\sim 80 \mathrm{MV} \mathrm{m}^{-1}\right)$.

Highly dipolar sulfonyl side groups are also used in DG polymers because of the high dipole moment of $4.5 \mathrm{D}$. Different from main-chain sulfonyl polymers, ${ }^{30,31}$ sulfonylated side chain polymers are more advantageous to enhance the dielectric constant because of the high mobility of side chain dipoles. For example, sulfonyl-containing polymethacrylates ${ }^{32,33}$ and polyethers $^{34}$ exhibited high $\kappa$ values between 7 and 12. However, they again suffer from relatively low $T_{\mathrm{g}}\left(<125{ }^{\circ} \mathrm{C}\right)$. Most recently, sulfonylated poly(2,6-dimethyl-1,4-phenylene oxide)s ( $\mathrm{SO}_{2}$-PPOs) with a rigid polymer backbone and high $T_{\mathrm{g}}$ above
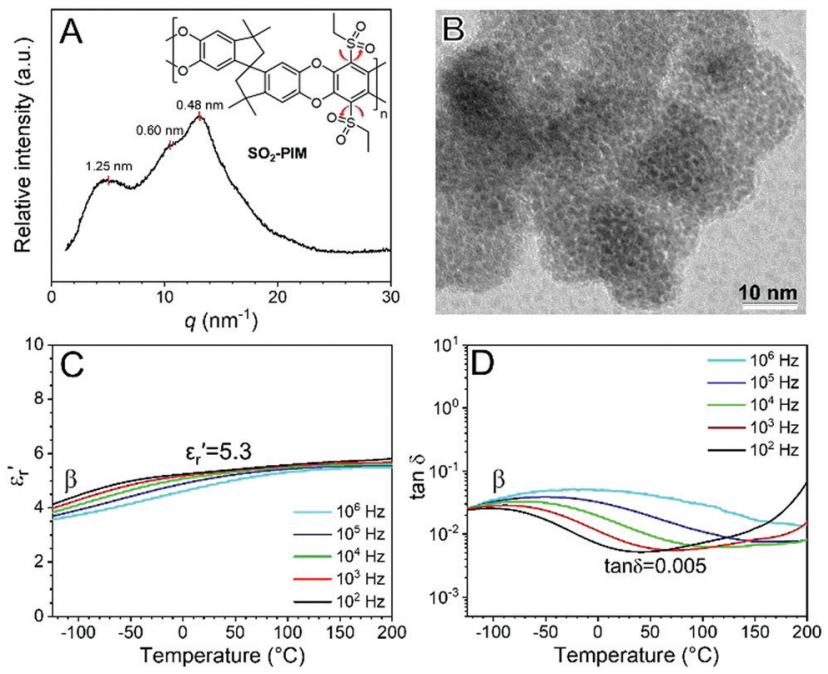

Fig. 1 (A) One-dimensional WAXD profile for the as-cast $\mathrm{SO}_{2}$-PIM (the chemical structure is in the inset). (B) Bright-field TEM micrograph of $\mathrm{SO}_{2}-$ PIM stained by $\mathrm{RuO}_{4}$ for $10 \mathrm{~min}$. Temperature-scan BDS results of (C) the real part of the relative permittivity $\left(\varepsilon_{\mathrm{r}}{ }^{\prime}\right)$ and (D) $\tan \delta$ at different frequencies.

$200{ }^{\circ} \mathrm{C}$ was reported. ${ }^{35}$ They demonstrated a high $\kappa$ of $6-8$, a relatively low dielectric loss, and heat resistence up to $150{ }^{\circ} \mathrm{C}$.

In this work, we report for the first time that polymers of intrinsic microporosity (PIMs) can be used as a new class of DG polymers for high-performance FETs. With a large free volume, rigid and contorted ladder-like backbones, and high $T_{\mathrm{g}}$ (above the thermal degradation temperature), high- $\kappa$ and low-loss PIMs are realized by attaching dipolar sulfonyl groups to the backbone of an organosoluble PIM ( $\mathrm{SO}_{2}$-PIM, see the inset in Fig. 1A). ${ }^{36}$ This could be attributed to the friction-free rotation of sulfonyl dipoles in the nanopores. A high $\kappa$ of 5-6 was obtained and the discharged energy density $\left(U_{\mathrm{e}}\right)$ was as high as $\sim 17 \mathrm{~J} \mathrm{~cm}^{-3}$ with the dissipation loss as low as $\tan \delta \sim 0.005$. More importantly, the $\mathrm{SO}_{2}$-PIM exhibited a high discharge efficiency of $94 \%$ at $150{ }^{\circ} \mathrm{C}$ and $300 \mathrm{MV} \mathrm{m}^{-1}$, demonstrating the outstanding heat resistance. Finally, its application as a high- $\kappa$ gate dielectric in the InSe FETs was verified, and an enhanced electron mobility of 200-400 $\mathrm{cm}^{2} \mathrm{~V}^{-1} \mathrm{~s}^{-1}$ was achieved.

Scheme S1 in the ESI $\dagger$ shows the modified synthesis of $\mathrm{SO}_{2}$-PIM. It was reported that high monomer concentration $(>25 \%)$ and high temperature $\left(160{ }^{\circ} \mathrm{C}\right)$ led to a high molecular weight for PIM-1, because a short reaction time could prevent crosslinking. ${ }^{36}$ However, the $\mathrm{Ar}-\mathrm{SO}_{2} \mathrm{CH}_{2} \mathrm{CH}_{3}$ group was too acidic because of strong electron withdrawing ability of the sulfonyl group, and it could react readily with the base $\left(\mathrm{K}_{2} \mathrm{CO}_{3}\right)$ to generate a crosslinked structure (Scheme S2, ESI $\dagger$ ). To avoid crosslinking, the disulfinyl monomer was used for the condensation polymerization instead of the disulfonyl monomer, because the electron withdrawing ability of the sulfinyl group is weaker. The condensation polymerization was stopped after $80 \mathrm{~min}$ at $160{ }^{\circ} \mathrm{C}$, and no crosslinking was observed. Then, the obtained SO-PIM was fully oxidized using $m$-chloroperbenzoic acid ( $m$-CPBA) in chloroform to afford $\mathrm{SO}_{2}$-PIM. After slow precipitation in acetone, low molecular weight fractions were removed. 
The molecular weight and molecular weight distribution of the purified polymer were determined by size-exclusion chromatography (SEC) using $N, N$-dimethylformamide (DMF) as a solvent and polystyrene as a standard. The number-average molecular weight $\left(M_{\mathrm{n}}\right)$ was $360000 \mathrm{Da}$ and the molecular weight distribution was 3.3. The $\mathrm{SO}_{2}$-PIM was soluble in various organic solvents such as chloroform, tetrahydrofuran, DMF, $\mathrm{N}, \mathrm{N}$ dimethylacetamide (DMAc), and dimethylsulfoxide (DMSO). Using this revised synthetic method, high quality $\mathrm{SO}_{2}$-PIM films could be obtained by solution-casting for dielectric measurements. Proton nuclear magnetic resonance and SEC characterization results are presented in Fig. S1-S3 (ESI $\dagger$ ).

Films of $\mathrm{SO}_{2}$-PIM $(25-30 \mu \mathrm{m})$ were obtained by solutioncasting from chloroform. Wide-angle X-ray Diffraction (WAXD) showed multiple broad peaks, corresponding to different pore sizes (0.5-1.25 nm) (Fig. 1A). The pore morphology was studied by transmission electron microscopy (TEM); see Fig. 1B. The $\mathrm{SO}_{2}$-PIM was stained by $\mathrm{RuO}_{4}$ vapor for $10 \mathrm{~min}$. Since $\mathrm{RuO}_{4}$ preferentially stained aromatic rings, the polymer struts appeared dark and the pores appeared brighter. From the TEM projection image, the average pore width was $c a .0 .6 \mathrm{~nm}$ with a run length of 1.2-1.5 nm, which fit with the WAXD result well. These nanopores provided large free volume for frictionfree rotation of the sulfonyl dipoles. The linear dielectric property was studied by conventional broadband dielectric spectroscopy (BDS). In Fig. 1C, the dielectric constant (i.e., the real part of relative permittivity, $\varepsilon_{\mathrm{r}}{ }^{\prime}$ ) at $1 \mathrm{kHz}$ increased from 4.0 at $-125{ }^{\circ} \mathrm{C}$ to 5.3 at room temperature, owing to the orientational polarization of two ethylsulfonyl side groups. The dipolar relaxation could be clearly seen in $\tan \delta$ (Fig. 1D) and the $\varepsilon_{\mathrm{r}}^{\prime \prime}$ (i.e., the imaginary part of relative permittivity, Fig. S4, ESI $\dagger$ ). The sub- $T_{\mathrm{g}}($ i.e., $\beta)$ transition took place around $-75^{\circ} \mathrm{C}$ at $1 \mathrm{kHz}$, and was assigned to the rotation of ethylsulfonyl side groups in the free volume. The frequency-scan BDS results for $\mathrm{SO}_{2}$-PIM at different temperatures are shown in Fig. S5 (ESI $\dagger$ ). The peak $\beta$ transition could be observed at high frequencies, i.e., $>10^{6} \mathrm{~Hz}$, when the temperature was above $50{ }^{\circ} \mathrm{C}$. Meanwhile, the lowest $\tan \delta$ reached as low as 0.005 for the $\mathrm{SO}_{2}$-PIM around $40{ }^{\circ} \mathrm{C}$ and $1 \mathrm{kHz}$. This was a result of the high $T_{\mathrm{g}}$ and thus largely frozen chain dynamics. The high-field dielectric behavior of $\mathrm{SO}_{2}$-PIM was further studied using high voltage BDS (HV-BDS). ${ }^{37}$ In Fig. S6 (ESI $\dagger$ ), all $\tan \delta$ curves under different electric fields largely overlapped, except for slightly enhanced conduction of impurity ions above $50{ }^{\circ} \mathrm{C}$. This result indicated that no significant dielectric nonlinearity was observed up to $71.4 \mathrm{MV} \mathrm{m}^{-1}$. This was different from main-chain dipolar glass polymers reported recently, ${ }^{37}$ where significant dielectric loss was observed as a result of high field-induced cooperative segmental motions.

The high-field dielectric property was studied by unipolar $D-E$ loop measurements, and discharged $U_{\mathrm{e}}$ and efficiency were obtained from the charge and discharge curves (Fig. 2). At $1 \mathrm{kHz}$, extremely narrow loops were obtained below $450 \mathrm{MV} \mathrm{m}^{-1}$ (Fig. 2A) with a discharge efficiency above 97\% (Fig. 2B), indicating that the sulfonyl dipoles were isolated and did not form any ferroelectric domains under the high poling field.
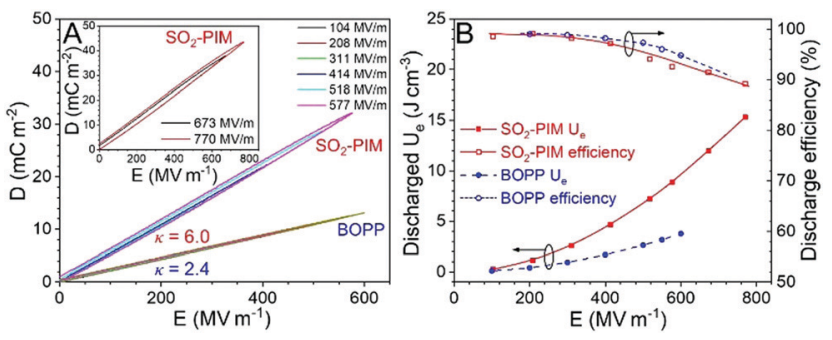

Fig. 2 (A) Unipolar $D-E$ loops for $\mathrm{SO}_{2}$-PIM under $1 \mathrm{kHz}$ at room temperature. (B) Discharged energy density $\left(U_{\mathrm{e}}\right)$ and discharge efficiencies as a function of the poling field for $\mathrm{SO}_{2}$-PIM. BOPP is used for comparison.

This is different from low- $T_{\mathrm{g}}\left(<125{ }^{\circ} \mathrm{C}\right)$ sulfone-containing dipolar glass polymers, ${ }^{34}$ where weak ferroelectric domains could still form under a high enough electric field $\left(>400 \mathrm{MV} \mathrm{m}^{-1}\right)$. A high $\kappa$ of 6.0 was obtained from the slope of the linear loops.

Compared with the state-of-the-art BOPP film, the discharge efficiency was similar; however, the discharged $U_{\mathrm{e}}$ of $\mathrm{SO}_{2}$-PIM was about 2.5 times that of BOPP. When the poling field increased to above $500 \mathrm{MV} \mathrm{m}^{-1}$, dielectric loss became more pronounced (Fig. 2A), which could be attributed to enhanced electronic conduction under high electric fields. ${ }^{38}$ However, the discharge efficiency was still $\geq 90 \%$ (Fig. 2B). When the poling field reached $770 \mathrm{MV} \mathrm{m}^{-1}$, a high discharged $U_{\mathrm{e}}$ of $17 \mathrm{~J} \mathrm{~cm}^{-3}$ was achieved.

Similar to other reports, ${ }^{36}$ no glass transition was found for $\mathrm{SO}_{2}$-PIM before the onset of thermal degradation (i.e., $360{ }^{\circ} \mathrm{C}$ with $1 \mathrm{wt} \%$ weight loss). The exceptionally high $T_{\mathrm{g}}$ of PIMs is desired for high-temperature dielectric applications. Fig. 3A shows high temperature bipolar $D-E$ loops for the $\mathrm{SO}_{2}$-PIM film polarized at $300 \mathrm{MV} \mathrm{m}^{-1}(1 \mathrm{kHz})$. Below $150{ }^{\circ} \mathrm{C}$, slim loops were observed. Fig. 3B presents the discharged $U_{\mathrm{e}}$ and discharge efficiency for $\mathrm{SO}_{2}$-PIM as a function of temperature, and the recently reported $\mathrm{SO}_{2}-\mathrm{PPO}_{25}$ was used for comparison. ${ }^{35}$ At $100{ }^{\circ} \mathrm{C}, \mathrm{SO}_{2}-\mathrm{PPO}_{25}$ exhibited a higher discharged $U_{\mathrm{e}}$ than $\mathrm{SO}_{2}$ PIM because of its higher $\kappa$ of 6.2. However, $\mathrm{SO}_{2}$-PIM had a higher discharge efficiency (94.4\%) than that of $\mathrm{SO}_{2}-\mathrm{PPO}_{25}$ (91\%). Upon increasing the temperature to $150{ }^{\circ} \mathrm{C}$, the discharged $U_{\mathrm{e}}$ of $\mathrm{SO}_{2}$-PIM remained nearly constant and the discharge efficiency only slightly decreased to $93 \%$. However, both the discharged $U_{\mathrm{e}}$ and discharge efficiency of $\mathrm{SO}_{2}-\mathrm{PPO}_{25}$ significantly decreased upon increasing the temperature to $150{ }^{\circ} \mathrm{C}$. This was explained by enhanced charge injection from electrodes and thus high electronic conduction at elevated temperatures. ${ }^{35}$ Considering similar dielectric constants, it should be the higher $T_{\mathrm{g}}$ and suppressed space charge injection from metal electrodes for $\mathrm{SO}_{2}$-PIM to exhibit a lower dielectric loss than $\mathrm{SO}_{2}-\mathrm{PPO}_{25}$. Even at $175{ }^{\circ} \mathrm{C}$, the $\mathrm{SO}_{2}$-PIM film still exhibited a reasonable discharge efficiency of $86 \%$. When the poling field decreased to $200 \mathrm{MV} \mathrm{m}^{-1}$, high discharge efficiencies were still observed, i.e., $94 \%$ at $175{ }^{\circ} \mathrm{C}$ and $88 \%$ at $200{ }^{\circ} \mathrm{C}$ (Fig. 3C). These high temperature discharge efficiencies were comparable to a crossliniked divinyltetramethyldisiloxanebis(benzocyclo-butene)/boron nitride nanosheet (c-BCB/BNNS) composite reported recently. ${ }^{39}$ However, the discharged $U_{\mathrm{e}}$ of $\mathrm{SO}_{2}$-PIM 

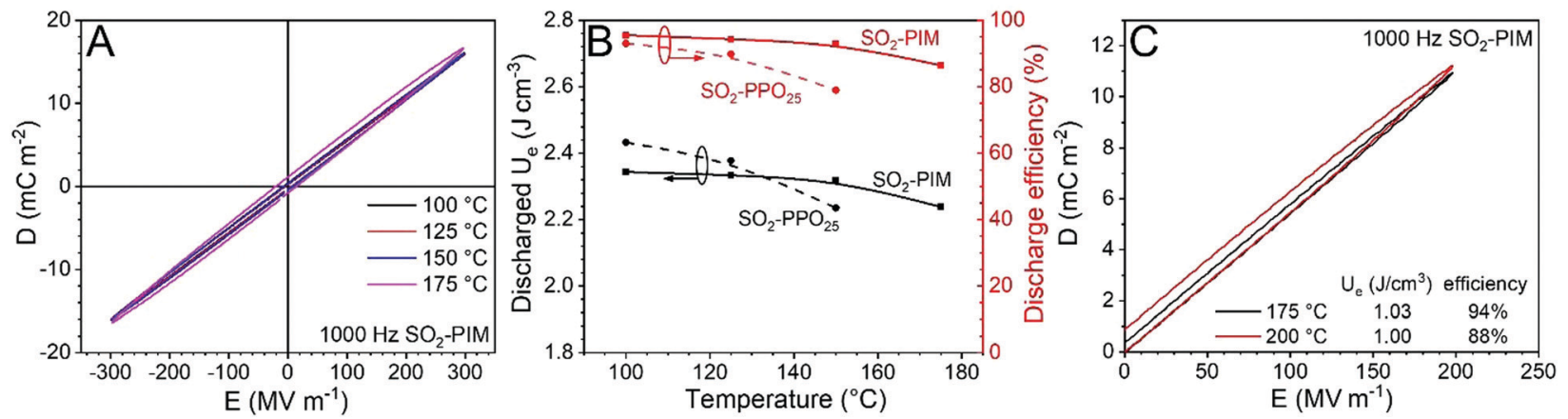

Fig. 3 (A) Bipolar $D-E$ loops at high temperatures for $\mathrm{SO}_{2}-\mathrm{PIM}$. (B) Tempearture-dependent discharged energy density $\left(U_{\mathrm{e}}\right)$ and discharge efficiency for $\mathrm{SO}_{2}-\mathrm{PIM}$ and $\mathrm{SO}_{2}$-PPO under $300 \mathrm{MV} \mathrm{m}^{-1}$. (C) Unipolar D-E loops under $200 \mathrm{MV} \mathrm{m}^{-1}$ for SO $\mathrm{S}_{2}-\mathrm{PIM}$ at 175 and $200{ }^{\circ} \mathrm{C}$, respectively.

was about twice that of $\mathrm{c}-\mathrm{BCB} / \mathrm{BNNS}$ because of its higher dielectric constant, i.e., 6.0 for $\mathrm{SO}_{2}$-PIM vs. 3.1 for $\mathrm{c}-\mathrm{BCB} / \mathrm{BNNS}$.

Finally, the $\mathrm{SO}_{2}$-PIM was applied as a high- $\kappa$ gate dielectric for InSe-based FETs. InSe is a 2D semiconductor with high electron mobility as well as strong spin-orbit coupling and has drawn a lot of attention in the past few years for the fabrication of high performance 2D semiconductor FETs or transport devices. ${ }^{40-42}$ As shown in Fig. 4A, an InSe nanoflake $(\sim 20 \mathrm{~nm}$ thick) was exfoliated onto a $\mathrm{Si} / \mathrm{SiO}_{2}$ substrate coated with $\mathrm{SO}_{2}$-PIM where both the silicon oxide $(300 \mathrm{~nm})$ and $\mathrm{SO}_{2}$-PIM $(300-800 \mathrm{~nm})$ were used as the gate dielectric. Electron beam evaporation was used to deposit a $5 \mathrm{~nm}$ layer of Ti followed by a $40 \mathrm{~nm}$ layer of $\mathrm{Ni}$ to form the source and drain electrodes contacting the InSe nanoflake on the $\mathrm{SO}_{2}$-PIM layer. The degenerately doped n-type Si substrate was used as the back-gate electrode. The InSe channel length was about $20 \mu \mathrm{m}$. To ensure good insulation, the leakage current density $(J)$ was tested for a solution-cast $\mathrm{SO}_{2}$-PIM thin film capacitor $(205 \mathrm{~nm})$

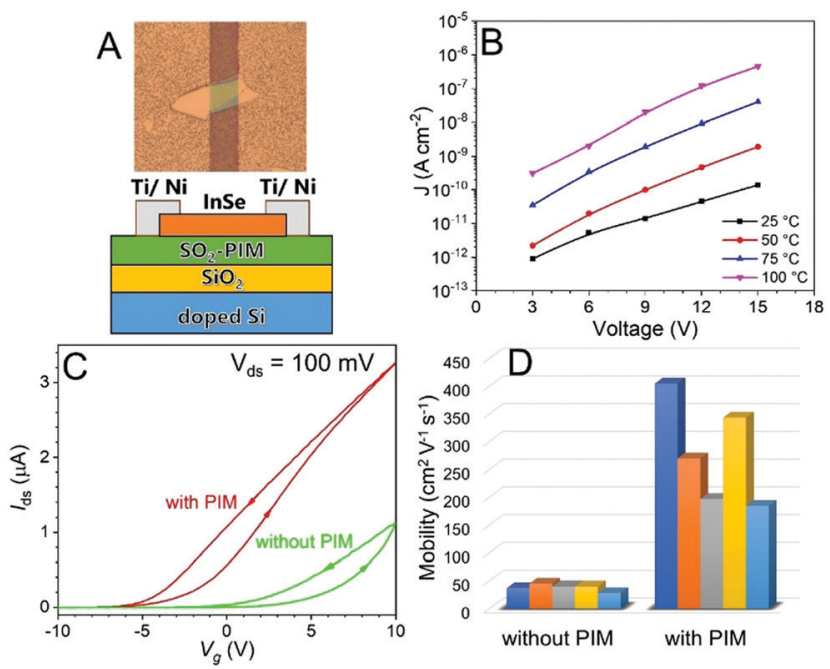

Fig. 4 (A) Schematic of the InSe-based FET device and the top-view optical microscopy image. (B) Leakage current density as a function of voltage in a capacitor device. (C) The $I_{\mathrm{ds}}-V_{\mathrm{g}}$ transfer curve and (D) comparison of electron mobility in InSe for the FET devices with and without $\mathrm{SO}_{2}$-PIM. Five devices were tested for each type of FET. at different temperatures. As seen in Fig. 4B, low $J$ values of $8.8 \times 10^{-13} \mathrm{~A} \mathrm{~cm}^{-2}$ at $3 \mathrm{~V}$ and $1.4 \times 10^{-10} \mathrm{~A} \mathrm{~cm}^{-2}$ at $15 \mathrm{~V}$ were observed for $\mathrm{SO}_{2}$-PIM at room temperature. At $100{ }^{\circ} \mathrm{C}$, the insulating property of $\mathrm{SO}_{2}$-PIM was still preserved with a relativley low $J$ value of $1.9 \times 10^{-9} \mathrm{~A} \mathrm{~cm}^{-2}$ at $6 \mathrm{~V}$.

Typical $I_{\mathrm{ds}}-V_{\mathrm{g}}$ transfer curves are shown in Fig. 4C for the FET devices with and without $\mathrm{SO}_{2}$-PIM. The electron's field effect mobility could be calculated from the linear region of the transfer curves. Note that the hysteresis in the transfer curves could be attributed to certain surface defects on the dielectric layer that allowed certain charge injection. ${ }^{43}$ All FET devices with $\mathrm{SO}_{2}$-PIM exhibited a high electron mobility between 180 and $400 \mathrm{~cm}^{2} \mathrm{~V}^{-1} \mathrm{~s}^{-1}$, about $10 \times$ higher than those for the FET devices without $\mathrm{SO}_{2}$-PIM (Fig. 4D). The enhancement of mobility could be attributed to the screening effect from high- $\kappa$ polar polymers. ${ }^{40,44}$ Namely, the $\mathrm{SO}_{2}$-PIM layer was used for screening interfacial Coulomb impurities, such as hydroxyl groups, moisture, and other chemical absorptions, resulting in improved charge carrier mobility and better performance of InSe FETs. Furthermore, $I_{\mathrm{ds}}-V_{\mathrm{g}}$ transfer curves in the logarithmic scale and $I_{\mathrm{ds}}-V_{\mathrm{ds}}$ curves are shown in Fig. S7 (ESI $\dagger$ ), demonstrating more clearly the ON-OFF switching of the device and ohmic behavior of the contacts. It can be seen in Fig. S7 (ESI $\dagger$ ) that there was an indication of improved ON-OFF ratio with the presence of the $\mathrm{SO}_{2}$-PIM dielectric in the InSe FET, and the ON-OFF ratio reached at least $10^{4}$ with the $\mathrm{SO}_{2}$ PIM gate dielectric (the actual ON-OFF ratio should be higher than $10^{4}$ shown in Fig. S7 (ESI $\dagger$ ), since the true OFF state was not reached in the measurement due to the limited sensitivity of the instrument). Combining relatively high dielectric constant, low dielectric loss, low leakage, and high-temperature tolerance, dipolar $\mathrm{SO}_{2}$-PIM is promising as a polymer gate dielectric for high-performance FETs.

In summary, utilizing highly polarizable sulfonyl groups, we introduced a new class of high- $\kappa$ (5.3-6.0), high temperature (up to $200{ }^{\circ} \mathrm{C}$ ), and low loss $(\tan \delta \sim 0.005)$ dielectric, $\mathrm{SO}_{2}$-PIM. The large pores $(0.5-1.25 \mathrm{~nm})$ were beneficial for the frictionfree rotation of sulfonyl side groups. The highly rigid polymer structure with a high $T_{\mathrm{g}}$ prevented the backbone motions, resulting in low leakage current, high breakdown strength, and low dielectric loss at high temperatures. At room temperature, 
the discharged $U_{\mathrm{e}}$ reached $17 \mathrm{~J} \mathrm{~cm}^{-3}$ at $770 \mathrm{MV} \mathrm{m}^{-1}$. When it was used as a high- $\kappa$ gate dielectric in the InSe FET device, a high electron mobility of $180-400 \mathrm{~cm}^{2} \mathrm{~V}^{-1} \mathrm{~s}^{-1}$ was achieved. These experimental results demonstrated exciting prospects for the polar PIMs to be used as high-performance dielectrics in printed electronics.

\section{Conflicts of interest}

The authors declare no competing financial interests.

\section{Acknowledgements}

This work is supported by the National Science Foundation, Division of Materials Research, Polymers (DMR-1708990) and Condensed Matter Physics (DMR-1607631) Programs. This research used the 11-BM CMS beamline of National Synchrotron Light Source-II, Brookhaven National Laboratory (BNL), a U.S. Department of Energy User Facility operated for the Office of Science under contract No. DE-SC0012704.

\section{References}

1 L. Zhu, J. Phys. Chem. Lett., 2014, 5, 3677-3687.

2 Prateek, V. K. Thakur and R. K. Gupta, Chem. Rev., 2016, 116, 4260-4317.

3 E. Baer and L. Zhu, Macromolecules, 2017, 50, 2239-2256.

4 G. Wang, N. Persson, P. H. Chu, N. Kleinhenz, B. Y. Fu, M. Chang, N. Deb, Y. M. Mao, H. Z. Wang, M. A. Grover and E. Reichmanis, ACS Nano, 2015, 9, 8220-8230.

5 G. Wang, W. Huang, N. D. Eastham, S. Fabiano, E. F. Manley, L. Zeng, B. H. Wang, X. A. Zhang, Z. H. Chen, R. Li, R. P. H. Chang, L. X. Chen, M. J. Bedzyk, F. S. Melkonyan, A. Facchetti and T. J. Marks, Proc. Natl. Acad. Sci. U. S. A., 2017, 114, E10066-E10073.

6 Y. Khan, A. E. Ostfeld, C. M. Lochner, A. Pierre and A. C. Arias, Adv. Mater., 2016, 28, 4373-4395.

7 S. T. Han, Y. Zhou and V. A. L. Roy, Adv. Mater., 2013, 25, 5425-5449.

8 F. Carpi, D. De Rossi, R. Kornbluh, R. E. Pelrine and P. Sommer-Larsen, Dielectric Elastomers as Electromechanical Transducers: Fundamentals, Materials, Devices, Models and Applications of an Emerging Electroactive Polymer Technology, Elsevier, Oxford, UK, 2008.

9 A. Facchetti, M. H. Yoon and T. J. Marks, Adv. Mater., 2005, 17, 1705-1725.

10 Y. G. Ha, K. Everaerts, M. C. Hersam and T. J. Marks, Acc. Chem. Res., 2014, 47, 1019-1028.

11 R. P. Ortiz, A. Facchetti and T. J. Marks, Chem. Rev., 2010, 110, 205-239.

12 Y. H. Chou, H. C. Chang, C. L. Liu and W. C. Chen, Polym. Chem., 2015, 6, 341-352.

13 S. A. DiBenedetto, A. Facchetti, M. A. Ratner and T. J. Marks, Adv. Mater., 2009, 21, 1407-1433.

14 A. A. Demkov and A. Navrotsky, Materials Fundamentals of Gate Dielectrics, Springer, Dordrecht, Netherland, 2005.
15 A. Gupta, P. Fang, M. Song, M. R. Lin, D. Wollesen, K. Chen and C. M. Hu, IEEE Electron Device Lett., 1997, 18, 580-582.

16 Q. Chen, Y. Shen, S. Zhang and Q. M. Zhang, Annu. Rev. Mater. Res., 2015, 45, 433-458.

17 C. Vericat, M. E. Vela, G. Benitez, P. Carro and R. C. Salvarezza, Chem. Soc. Rev., 2010, 39, 1805-1834.

18 R. L. McCreery and A. J. Bergren, Adv. Mater., 2009, 21, 4303-4322.

19 L. Yang, X. Li, E. Allahyarov, P. L. Taylor, Q. M. Zhang and L. Zhu, Polymer, 2013, 54, 1709-1728.

20 J. H. Li, Z. H. Sun and F. Yan, Adv. Mater., 2012, 24, 88-93.

21 X. Chen, L. Liu, S. Z. Liu, Y. S. Cui, X. Z. Chen, H. X. Ge and Q. D. Shen, Appl. Phys. Lett., 2013, 102, 063103.

22 J. O. M. Bockris, A. K. N. Reddy and M. Gamboa-Aldeco, Modern Electrochemistry 2A, Kluwer Academic Publishers, New York, 2nd edn, ch. 6, 2000.

23 S. H. Kim, K. Hong, W. Xie, K. H. Lee, S. Zhang, T. P. Lodge and C. D. Frisbie, Adv. Mater., 2013, 25, 1822-1846.

24 J. R. Fried, in Physical Properties of Polymers Handbook, ed. J. E. Mark, Springer, New York, 2007.

25 R. H. M. Vandeleur, Polymer, 1994, 35, 2691-2700.

26 B. A. Bedekar, Y. Tsujii, N. Ide, Y. Kita, T. Fukuda and T. Miyamoto, Polymer, 1995, 36, 4735-4740.

27 T. Sato, Y. Tsujii, Y. Kita, T. Fukuda and T. Miyamoto, Macromolecules, 1991, 24, 4691-4697.

28 S. Tasaka, N. Inagaki, S. Miyata and T. Chiba, Sen'i Gakkaishi, 1988, 44, 546-550.

29 J. Y. Kim, H. Kim, T. Kim, S. Yu, J. W. Suk, T. Jeong, S. Song, M. J. Bae, I. Han, D. Jung and S. H. Park, J. Mater. Chem. C, 2013, 1, 5078-5083.

30 T. Zhang, M. H. Litt and C. E. Rogers, J. Polym. Sci., Part A: Polym. Chem., 1994, 32, 2809-2816.

31 T. Zhang, M. H. Litt and C. E. Rogers, J. Polym. Sci., Part A: Polym. Chem., 1994, 32, 2291-2300.

32 J. Wei, Z. Zhang, J.-K. Tseng, I. Treufeld, X. Liu, M. H. Litt and L. Zhu, ACS Appl. Mater. Interfaces, 2015, 7, 5248-5257.

33 Y. X. Wang, X. Y. Huang, T. Li, Z. W. Wang, L. Q. Li, X. J. Guo and P. K. Jiang, J. Mater. Chem. A, 2017, 5, 20737-20746.

34 Y.-F. Zhu, Z. Zhang, M. H. Litt and L. Zhu, Macromolecules, 2018, 51, 6257-6266.

35 Z. Zhang, D. H. Wang, M. H. Litt, L.-S. Tan and L. Zhu, Angew. Chem., Int. Ed., 2018, 57, 1528-1531.

36 N. Du, G. P. Robertson, I. Pinnau and M. D. Guiver, Macromolecules, 2009, 42, 6023-6030.

37 Z. Zhang, M. H. Litt and L. Zhu, Macromolecules, 2018, 51, 1967-1977.

38 S. Wu, W. Li, M. Lin, Q. Burlingame, Q. Chen, A. Payzant, K. Xiao and Q. M. Zhang, Adv. Mater., 2013, 25, 1734-1738.

39 Q. Li, L. Chen, M. R. Gadinski, S. Zhang, G. Zhang, H. U. Li, E. Iagodkine, A. Haque, L.-Q. Chen, T. N. Jackson and Q. Wang, Nature, 2015, 523, 576-579. 
40 S. Sucharitakul, N. J. Goble, U. R. Kumar, R. Sankar, Z. A. Bogorad, F. C. Chou, Y. T. Chen and X. P. A. Gao, Nano Lett., 2015, 15, 3815-3819.

41 K. Premasiri, S. K. Radha, S. Sucharitakul, U. R. Kumar, R. Sankar, F. C. Chou, Y. T. Chen and X. P. A. Gao, Nano Lett., 2018, 18, 4403-4408.

42 D. A. Bandurin, A. V. Tyurnina, G. L. Yu, A. Mishchenko, V. Zólyomi, S. V. Morozov, R. K. Kumar, R. V. Gorbachev,
Z. R. Kudrynskyi, S. Pezzini, Z. D. Kovalyuk, U. Zeitler, K. S. Novoselov, A. Patanè, L. Eaves, I. V. Grigorieva, V. I. Falko, A. K. Geim and Y. Cao, Nat. Nanotechnol., 2016, 12, 223-227.

43 H. Wang, Y. Wu, C. Cong, J. Shang and T. Yu, ACS Nano, 2010, 12, 7221-7228.

44 W. Feng, W. Zheng, W. Cao and P. Hu, Adv. Mater., 2014, 26, 6587-6593. 